\title{
Connectedness Based on Shared Engagement Predicts Remote Biochemically Verified Quit Status Within Smoking Cessation Treatment Groups on Facebook
}

\author{
Meredith C. Meacham PhD, MPH ${ }^{1,}$, Ou Stella Liang MHA ${ }^{2}$, Mengnan Zhao BS ${ }^{2}$, \\ Christopher C. Yang PhD², Johannes Thrul PhD ${ }^{3, \oplus}$, Danielle E. Ramo PhD ${ }^{1,4}$
}

'Department of Psychiatry and Weill Institute for Neurosciences, University of California San Francisco, San Francisco, CA; ${ }^{2}$ College of Computing and Informatics, Drexel University, Philadelphia, PA; ${ }^{3}$ Department of Mental Health, Johns Hopkins Bloomberg School of Public Health, Baltimore, MD; ${ }^{4}$ Hopelab, San Francisco, CA

Corresponding Author: Meredith C. Meacham PhD, MPH, Department of Psychiatry and Weill Institute for Neurosciences, University of California San Francisco, 350 Parnassus Ave, San Francisco, CA 94117, USA. Telephone: 628-206-6728;

Fax: 415-476-7320; E-mail: meredith.meacham@ucsf.edu

\begin{abstract}
Introduction: Engagement with online content and online social network integration are associated with smoking behavior change, but less is known about social dynamics of shared engagement between participants in group-based social media interventions.

Methods: Participants were 251 young adult smokers aged 18 to 25 assigned to one of 29 secret Facebook groups tailored to their readiness to quit smoking ("pre-contemplation," "contemplation," and "preparation"). Groups varied in size and were randomly assigned to receive monetary incentives for engagement. All groups received daily posts for 90 days and were assessed for remote biochemically verified smoking abstinence at the end of the intervention. Across 29 groups, we examined associations between group features (group size, incentive condition, readiness to quit) with how connected members were within the group based on shared engagement with the same content (measured by density). At the individual level, we examined associations between 7-day biochemically verified smoking abstinence and how connected an individual was within the group (measured by degree centrality).

Results: After adjusting for comment volume, being in a contemplation group (vs. precontemplation group) was associated with a decrease in comment-based density. Individual degree centrality was significantly associated with biochemically verified smoking abstinence for both comments and likes.

Conclusions: Future group-based social media interventions for smoking cessation may want to focus on promoting connected engagement between participants, rather than simply quantity of engagement.

Implications: Participants in a smoking cessation intervention delivered through Facebook groups were more likely to have biochemically verified smoking abstinence if they were more connected to the rest of the group via shared engagement. Promoting shared engagement between participants may be more likely to promote behavior change than volume of engagement alone.
\end{abstract}




\section{Introduction}

One in four young adults aged 18 to 25 in the United States report using tobacco in the past month ${ }^{1}$ and almost all smokers report starting before the age of $26 .{ }^{2}$ Young adult smokers underutilize available evidence-based treatments for quitting (eg, nicotine replacement therapy and counseling). ${ }^{3-5}$ However, $90 \%$ of young adults aged 18 to 29 report currently using social media, ${ }^{6}$ and Facebook remains the most popular platform in this age group, with $81 \%$ reporting current use and $74 \%$ reporting daily use in $2018 .^{6}$

Engagement with online and social media intervention content has been associated with behavior change. ${ }^{7-10}$ We recently demonstrated the short-term efficacy of the Tobacco Status Project, a Facebook smoking cessation intervention. ${ }^{11}$ We found that engagement with the 3-month intervention (operationalized as number of comments by each individual) was significantly related to readiness to quit smoking and whether a monetary incentive was received for commenting engagement. Specifically, comment volume was greater among those in pre-contemplation and preparation groups versus contemplation groups. Comment volume was also greater for those who received an incentive versus no incentive and was positively associated with group size. However, neither volume of comments nor receiving a monetary incentive predicted post-intervention smoking abstinence. ${ }^{12}$ Yet, in a smaller feasibility trial of the Tobacco Status Project, greater commenting was related to smoking abstinence. ${ }^{13}$ In another Facebook group-based intervention with 16 adult participants in one group, combined engagement and social support was associated with a reduction in smoking, ${ }^{14}$ and other social media cessation interventions have also reported positive associations between engagement and cessation outcomes. ${ }^{15,16}$ In sum, there is conflicting evidence whether or not engagement is associated with smoking cessation outcomes. This variation in findings may be explained by other ways of examining engagement than number of comments, such as how participants connect to each other in the group context.

Social ties are important influences in both tobacco smoking and cessation, ${ }^{17}$ through mechanisms of social support and information sharing. In online settings, connectedness within social networks has been associated with reported smoking abstinence among QuitNet online community members ${ }^{18}$ and inversely associated with length of smoking cessation in the National Cancer Institute's Facebook community. ${ }^{19}$ Tie formation among members was also associated with smoking abstinence in BecomeAnEx.org, an online cessation program. ${ }^{20}$ However, these findings examined large online social networks of hundreds or thousands of members in a single network (although smaller sub-networks may form organically). Previous research has indicated that online social networks typically have about $17-20$ active participants ${ }^{8}$ and that sensitive information is more likely to be transmitted via a limited number of "strong ties." ${ }^{11}$ Furthermore, as group size grows, members may become less likely to contribute or engage. ${ }^{22}$ To inform future intervention development, more research is needed on social network dynamics in social media smoking cessation interventions that contain multiple smaller groups segmented by design. In an online context at the group level, this includes how connected members were within the group based on shared engagement (measured by group density). At the individual level, this is measured by how connected an individual is within the group (individual degree centrality).

In this article, we use social network analyses to characterize shared engagement (ie, commenting or liking) of the same content among group members in a smoking cessation intervention delivered through Facebook. We then examine relationships between group size, monetary incentive, and readiness to quit with group density, and between individual degree centrality and individual smoking abstinence. Our hypotheses were that group features may influence shared engagement between group members and that greater shared engagement with other group members may be associated with smoking abstinence.

\section{Methods}

\section{Recruitment}

Participants were young adult smokers recruited through the Facebook advertising platform. ${ }^{11,23}$ Eligibility criteria included being 18 to 25 years old, English literate, smoking at least 1 cigarette a day at least 3 days a week, having smoked at least 100 cigarettes in their life, and using Facebook at least 4 days a week. Participants completed online informed consent and were randomized to the Facebook intervention treatment arm $(n=251,50.2 \%)$, or to the control arm, who received a referral to the National Cancer Institute cessation program smokefree.gov $(N=249,49.8 \%)$. University of California San Francisco Institutional Review Board approved study procedures.

\section{Group Assignment and Assessments}

Participants in the treatment group were block randomized to one of 29 secret Facebook groups based on daily smoking status and their Transtheoretical Mode ${ }^{24}$ readiness to quit smoking: precontemplation (9 groups), contemplation (11 groups), and preparation ( 9 groups). Groups varied in size from 3 to 18 members (mean group size $=8.6$ ). Groups were also randomly assigned to one of four monetary incentive conditions: none ( 7 groups), monthly ( 8 groups), weekly ( 8 groups), daily (6 groups). Participants in all groups assigned to an incentive condition received up to $\$ 90$ (ie, $\$ 30 /$ month, $\$ 7 /$ week, or $\$ 1 /$ day) over the 90 -day intervention period.

Survey assessments were conducted online at baseline and 3 months (ie, 90 days) and participants received \$20 gift cards for completing assessments. When participants reported in the online assessment that they had not smoked any cigarettes in the past 7 days, not even a puff, they were considered to have self-reported abstinence. These participants were then mailed a saliva cotinine test strip to biochemically assess abstinence, which has been recommended for smoking cessation intervention studies ${ }^{25}$ (remote assessment procedure described below). ${ }^{26}$

\section{Intervention}

Facebook groups received daily automated posts for 90 days. Posts were created by research staff and content was adapted from the US Clinical Practice Guidelines ${ }^{27}$ for smoking cessation and the Transtheoretical Model of behavior change. ${ }^{24}$ Post content included a question or a prompt to participants, which they were instructed to respond to as a comment. Participants could also "like" the post or comments from other participants. Weekly live sessions with a $\mathrm{PhD}$-level smoking cessation counselor were also available as part of the intervention.

\section{Individual and Social Network Measures}

Participant baseline measures included age, gender, race/ethnicity, cigarettes smoked per day, daily smoking (yes/no), and readiness to quit smoking (ie, Transtheoretical Model stage of change). At the end of the intervention ( 3 months), participants self-reported 
whether they had smoked at all in the past 7 days. If they reported abstinence, they were mailed a cotinine saliva test kit and asked to send a photo of the test strip back to study staff via email or Facebook message. ${ }^{26}$ Test results indicating $<11 \mathrm{ng} / \mathrm{ml}$ cotinine were classified as biochemically confirmed abstinence, as were test results with $11-30 \mathrm{ng} / \mathrm{ml}$ cotinine if participants also reported e-cigarette use. Those who did not return their test kit or had results indicate cotinine exposure were classified as not biochemically verified abstinent.

Two undirected social networks (one based on comments and one based on likes) were created. We examined both comment and like networks because participants were instructed and incentivized to comment on posts daily, yet liking is still a form of engagement that signals receipt or approval of content. Then one group-level and one individual-level social network metric were calculated, where a connection or tie " $C$ " exists when two members of the Facebook group have engaged with (commented on or liked) the same content and $N$ is the total number of group members: ${ }^{28}$

Density (group-level) is the proportion of actual connection ties $(C)$ over total number of possible connections in a network of $N$ nodes (ie, the proportion of connections between members of the same group who engaged or co-participated with the same content $(2 \mathrm{C})$ out of the total possible connections ( $N$ $\times(N-1))$.

$$
\text { Density }=\frac{2 C}{N \times(N-1)}
$$

For example, if a group of 9 members had 18 connections or ties from members commenting on the same content, then density is $.50(2 * 18 /(9 *(9-1)))$. In an undirected network, the number of connections are multiplied by 2 to account for both people in the connection.

Degree centrality (individual-level) measures the number of connections a person has $\left(C_{\mathrm{i}}\right)$ over the total number of possible connections for that person (ie, The number of other people one person has shared engagement or co-participated in the same content) with divided by the number of other people in the Facebook group (N-1).

$$
\text { Degree centrality }=\frac{C_{i}}{(N-1)}
$$

For example, if someone in a group of 9 members comments on the same posts as 5 other people, then their degree centrality is $.63(5 /(9-1)$.
In summary, density (group-level) represents the connectedness among all members in the group based on who engaged with the same content (with a possible range of 0 to 1 ). Degree centrality (individual-level) represents the connectedness of the individual within the group via engagement with the same content (with a possible range of 0 to 1 ) (Table 1 ).

\section{Analysis}

Data was acquired via the Facebook API and de-identified. Relevant attributes such as message content, commenter ID, and type of user activity (ie, comments versus likes) were extracted from the raw JSON file to form adjacency matrices using Python. One conversation thread consisted of an original post (user or staff-generated), and any follow-up comments or likes. In a conversation thread under the same post, the intended audience of a comment is usually ambiguous. Therefore, we assumed that users engaging in the same thread shared connections with one another indiscriminately. Moderators and counselors from the study group were excluded from forming the network.

First, we examined associations between features of the 29 groups (group size, incentive condition, readiness to quit) with how connected members were within the group (measured by density). Distributions of network metrics were assessed using a Shapiro-Wilk normality test and equal variance tests ( $\mathrm{F}$ test and Levene's test). Mean values for comment density were compared across readiness to quit groups (using analysis of variance [ANOVA]) and incentive condition (yes/no) (using unpaired two-sample $t$ test). Median values for like-based density were compared across readiness to quit groups (using Kruskal Wallis test) and incentive condition (yes/no) (using Wilcoxon rank sum test). Pearson correlations were calculated to assess relationships between group size and density.

Linear regression models were estimated with density as the dependent variable and group features as the independent variables. Since there may be more opportunities for shared engagement to take place when there are more comments or likes, we also examined whether volume of engagement confounded the relationship between group features and social network metrics, via inclusion of this factor in multivariate regression models. Statistical analyses were performed in $\mathrm{R}$ version 3.4.3.

Finally, we examined individual-level associations between 7-day smoking abstinence (available for 169 participants) and how connected an individual was within the group (measured by degree centrality). Median degree centrality was compared between those who were abstinent versus not abstinent at the end of the 90-day intervention (self-reported and biochemically verified abstinence). Since abstinence was associated with readiness to quit and incentive condition as well as degree centrality, logistic regression analyses (with

\begin{tabular}{|c|c|c|}
\hline Term & Definition & Measurement \\
\hline Engagement & $\begin{array}{l}\text { Commenting on or liking study content posted in a } \\
\text { secret Facebook group }\end{array}$ & Number of comments or likes \\
\hline Shared engagement & $\begin{array}{l}\text { Commenting or liking the same content as another } \\
\text { Facebook group member }\end{array}$ & -- \\
\hline Density & $\begin{array}{l}\text { Connectedness among all members in the group based } \\
\text { on who engaged with the same content }\end{array}$ & $\begin{array}{l}\text { Proportion of shared engagement connections out of the total } \\
\text { possible connections within Facebook group }\end{array}$ \\
\hline Degree centrality & $\begin{array}{l}\text { Connectedness of the individual within the group via } \\
\text { engagement with the same content }\end{array}$ & $\begin{array}{l}\text { Number of shared engagement connections an individual } \\
\text { has out of total possible individual connections within } \\
\text { Facebook group }\end{array}$ \\
\hline
\end{tabular}

Table 1. Shared Engagement and Social Network Connection Definitions 


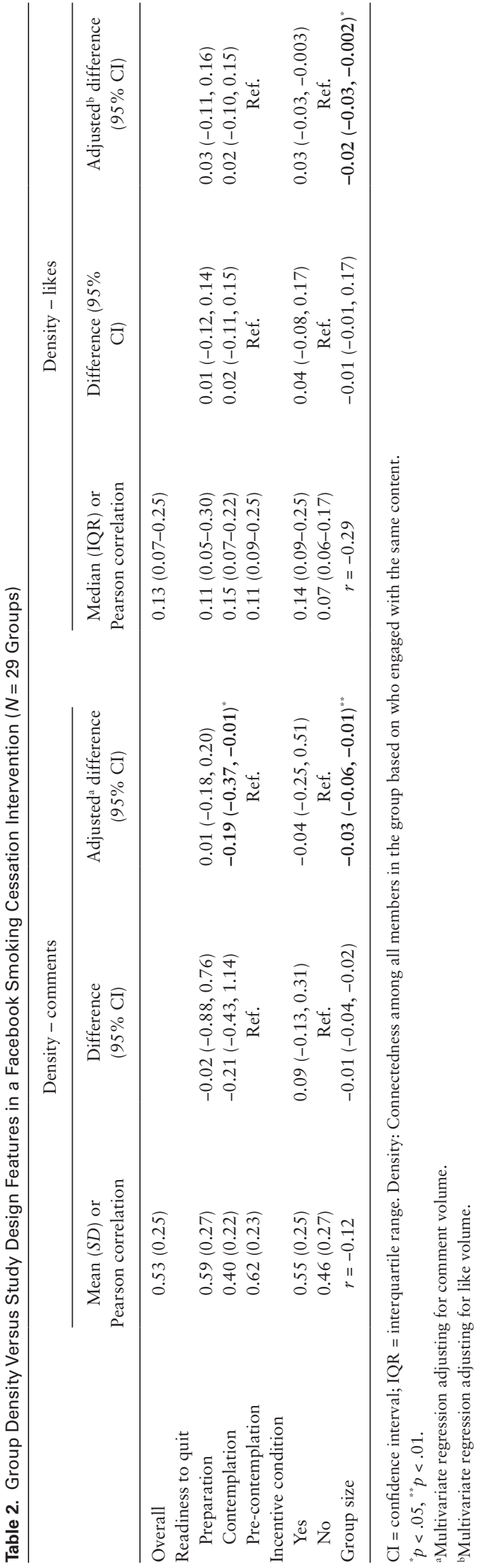

abstinence as dependent variable) adjusted for readiness to quit and incentive condition.

\section{Results}

Participants $(N=251)$ were $55 \%$ female and $77 \%$ non-Hispanic Caucasian with a mean age of $20.9(S D=2.0)$. At baseline, participants reported smoking on average 10.8 cigarettes per day $(S D=6.3)$ and $87.3 \%$ reported being daily smokers. Of 250 participants with Facebook data (one participant left Facebook at the start of the study), $82 \%(N=205)$ commented on or liked content at least once; $76 \%(N=192)$ commented at least once and $56.8 \%$ $(N=142)$ liked content at least once. The median number of comments by an individual was 13 (interquartile range [IQR]: 1-66) for the full sample and 31 (IQR: 7-84) for those who commented at least once. ${ }^{12}$ The median number of likes by an individual was 1 (IQR: 0-3) for the full sample and 2.5 (IQR: 1-5) for those who clicked "like" at least once. At the end of the 3-month intervention, $68 \%$ of participants $(N=169)$ completed follow-up surveys and reported whether they had abstained from smoking in the past 7 days.

\section{Group-Level Findings}

Mean density for comments was $0.53(S D=0.25)$. Like-based density was non-normally distributed, with a median density of 0.13 (IQR: 0.07-0.25). Neither readiness to quit nor incentive condition (yes/no) was significantly associated with density. There were no significant differences with regard to the frequency of incentive and density, either (Table 2).

\section{Adjusted Group-Level Findings}

Although comment-based density was not associated with any group features, after adjusting for comment volume, density for comments was negatively associated with contemplation compared to precontemplation (adjusted difference $=-0.19,95 \%$ confidence interval (CI): $-0.37,-0.01)$. In other words, after adjusting for number of comments in the group, the connectedness among all members decreased by 0.19 for contemplation groups versus pre-contemplation groups. After adjusting for comment volumes, comment-based density decreased by 0.03 (95\% CI: $-0.06,-0.01)$ for each additional member in a group.

There was no significant difference in like-based engagement measures between groups with different features, other than that after adjusting for like volume, like-based density decreased by 0.02 (95\% CI: $-0.03,-0.002$ ) for each additional group member.

\section{Individual-Level Findings}

Of 169 participants who completed a 3-month follow up survey, $13.6 \%(n=23)$ self-reported 7-day abstinence. These 23 participants were mailed a saliva cotinine test kit and $8.3 \%(n=14)$ of the 169 participants (or $60.9 \%$ of the 23 participants who reported abstinence) were biochemically verified abstinent via their returned test kit. Those with self-reported but not biochemically confirmed abstinence either did not return the kit results or had high cotinine levels due to having smoked between the online survey and taking the cotinine test or frequent vaping. In a previous analysis, we found that there were no significant differences with respect to sociodemographic factors, smoking history, dependence, or goals between those who reported abstinence and those who returned their test kits. ${ }^{26}$ 
Table 3. Individual Degree Centrality and Smoking Abstinence in a Facebook Smoking Cessation Intervention ( $N=169$ Participants Who Completed End-of-Treatment Assessment)

\begin{tabular}{|c|c|c|c|c|c|c|}
\hline & \multicolumn{3}{|c|}{ Degree centrality - comments } & \multicolumn{3}{|c|}{ Degree centrality - likes } \\
\hline & Median (IQR) & $\begin{array}{l}\text { Odds ratio } \\
(95 \% \mathrm{CI})\end{array}$ & $\begin{array}{c}\text { Adjusted }^{a} \text { odds ratio } \\
(95 \% \mathrm{CI})\end{array}$ & Median (IQR) & $\begin{array}{l}\text { Odds ratio } \\
(95 \% \mathrm{CI})\end{array}$ & $\begin{array}{l}\text { Adjusted }{ }^{a} \text { odds } \\
\text { ratio }(95 \% \mathrm{CI})\end{array}$ \\
\hline $\begin{array}{l}\text { Overall } \\
\text { Biochemically verified } \\
\text { abstinent }\end{array}$ & $0.67(0.38-0.80)$ & & & $0.13(0.00-0.30)$ & & \\
\hline Yes $(n=14)^{\mathrm{b}}$ & $0.80(0.71-0.96)$ & $9.70(1.16,152.0)^{*}$ & $16.38(1.43,406.9)^{*}$ & $0.25(0.17-0.40)$ & $11.15(0.99,122.9)$ & $22.53(1.58,378.3)^{*}$ \\
\hline No $(n=155)$ & $0.67(0.35-0.80)$ & Ref & Ref & $0.12(0.00-0.30)$ & Ref & Ref \\
\hline
\end{tabular}

$\mathrm{CI}=$ confidence interval; $\mathrm{IQR}=$ interquartile range.

*p $p .05$.

${ }^{\mathrm{a}}$ Multivariate regression adjusted for readiness to quit and incentive condition.

bIncludes two participants who reported e-cigarette use but tested under $30 \mathrm{ng} / \mathrm{ml}$ cotinine exposure.

Participants who were biochemically verified abstinent had a significantly higher median degree centrality (comment-based: 0.80, like-based: 0.25 ) than those who were not abstinent based on biochemical verification (comment-based: 0.67, like-based: 0.10) (Wilcoxon ran sum $W=710.5, p=.03$ for comments; Wilcoxon ran sum $W=732, p=.04$ for likes) (Table 2 ). There were no significant differences for self-reported abstinence, though median differences trended in a similar direction. In logistic regression analyses, the odds of verified abstinence were greater for those with higher degree centrality for both comments and likes, even after adjusting for individual-level readiness to quit and incentive condition (comments adjusted odds ratio $=16.38$ [95\% CI: 1.43, 406.9]; likes adjusted odds ratio $=22.53$ [95\% CI: $1.58,378.3$ ]; Table 3).

\section{Discussion}

In this investigation of social networks based on shared engagement, or co-participation, in a Facebook smoking cessation intervention with 250 individuals in 29 groups, we found that greater connectedness within the group via shared engagement (ie, degree centrality) was significantly associated with biochemically verified abstinence at the individual level. At the group level, after controlling for group comment volume, we found that contemplation groups had less overall connectedness between group members (ie, density) than those in pre-contemplation groups.

Participants who were more connected to other group members via shared engagement were significantly more likely to be biochemically verified abstinent, suggesting that individuals who share engagement experiences with others in the group may be more likely to change their behavior, regardless of their initial readiness to quit or having been incentivized to comment. A previous examination of centrality in a large Facebook smoking cessation group found that eigenvector centrality was inversely associated with length of cessation time, but that study did not examine centrality among those who were abstinent versus those who were not. ${ }^{19}$ Our finding of an association between shared engagement and biochemically verified abstinence across many smaller Facebook smoking cessation groups also supports the utility of group-based interventions ${ }^{29}$ that encourage group connectedness, dissemination and application of behavioral techniques, and mutual support in a social media setting.

The negative association of density with contemplation groups aligns with previous observations of low engagement in groups for participants in this stage of change category. ${ }^{12,13}$ This suggests merging these groups with participants in pre-contemplation, which aligns with previous research questioning the distinction between pre-contemplation and contemplation stages of change. ${ }^{30}$

There are several limitations to these findings. With 29 groups, we may have had reduced power to detect differences. We also do not know how much participants were likely to naturally engage with Facebook content outside of the intervention. Additionally, at the time of this intervention (2014-2015), participants could not consistently comment on other group members' comments, a feature that Facebook introduced later. Having these data would have allowed us to examine how engagement among participants relates to overall intervention engagement and smoking cessation. Finally, group members may still have viewed and thus passively "engaged" with content even if they did not comment or like the content. Nevertheless, this study is one of the first to look at the relationship between social network dynamics of engagement as they relate to biochemically verified smoking abstinence.

In summary, our findings demonstrate the utility of group-based interventions that facilitate shared engagement as leading to remote biochemically verified abstinence. Promoting shared engagement between participants may be more likely to promote behavior change than volume of engagement alone. Future interventions may want to collapse contemplation with pre-contemplation groups and to create content that promotes shared engagement.

\section{Funding}

This work was supported by the National Institute on Drug Abuse at the National Institutes of Health (grant numbers K23 DA032578, T32 DA007250, K01 DA046697).

\section{Declaration of Interests}

None declared.

\section{References}

1. SAMHSA. National Survey on Drug Use and Health. Rockville, MD: SAMHSA; 2017.

2. National Center for Chronic Disease Prevention and Health Promotion (US) Office on Smoking and Health. Preventing tobacco use among youth and young adults: A report of the surgeon general. In: Centers for Disease Control and Prevention (US), Atlanta, GA. 3, The epidemiology of tobacco 
use among young people in the united states and worldwide; 2012.https:// www.ncbi.nlm.nih.gov/books/NBK99243/.

3. Thrul J, Ramo DE. Cessation strategies young adult smokers use after participating in a Facebook Intervention. Subst Use Misuse. 2017;52(2):259-264.

4. Pinsker EA, Berg CJ, Nehl EJ, et al. Intent to quit among daily and nondaily college student smokers. Health Educ Res. 2013;28(2):313-325.

5. Watkins SL, Thrul J, Max W, Ling P. Cold Turkey and Hot Vapes? A national study of young adult cigarette cessation strategies. Nicotine Tob Res. 2020;22(5):638-646. doi:10.1093/ntr/nty270

6. Pew Research Center. Social media fact sheet. Fact Sheet. 2018. http:// www.pewinternet.org/fact-sheet/social-media/. Accessed May 7, 2018.

7. Hales SB, Davidson C, Turner-McGrievy GM. Varying social media post types differentially impacts engagement in a behavioral weight loss intervention. Transl Behav Med. 2014;4(4):355-362.

8. Pechmann C, Pan L, Delucchi K, Lakon CM, Prochaska JJ. Development of a Twitter-based intervention for smoking cessation that encourages high-quality social media interactions via automessages. J Med Internet Res. 2015;17(2):e50.

9. Strecher VJ, McClure J, Alexander G, et al. The role of engagement in a tailored web-based smoking cessation program: randomized controlled trial. J Med Internet Res. 2008;10(5):e36.

10. Schwarzer R, Satow L. Online intervention engagement predicts smoking cessation. Prev Med. 2012;55(3):233-236.

11. Ramo DE, Thrul J, Delucchi KL, et al. The Tobacco Status Project (TSP): Study protocol for a randomized controlled trial of a Facebook smoking cessation intervention for young adults. BMC Public Health. 2015;15:897.

12. Meacham MC, Ramo DE, Kral AH, Riley ED. Associations between medical cannabis and other drug use among unstably housed women. Int J Drug Policy. 2018;52:45-51.

13. Thrul J, Klein AB, Ramo DE. Smoking cessation intervention on Facebook: which content generates the best engagement? J Med Internet Res. 2015;17(11):e244.

14. Kim SJ, Marsch LA, Brunette MF, Dallery J. Harnessing Facebook for smoking reduction and cessation interventions: Facebook user engagement and social support predict smoking reduction. J Med Internet Res. 2017;19(5):e168.

15. Pechmann C, Delucchi K, Lakon CM, Prochaska JJ. Randomised controlled trial evaluation of Tweet2Quit: a social network quit-smoking intervention. Tob Control. 2017;26(2):188-194.
16. Graham AL, Papandonatos GD, Erar B, Stanton CA. Use of an online smoking cessation community promotes abstinence: results of propensity score weighting. Health Psychol. 2015;34S:1286-1295.

17. Christakis NA, Fowler JH. The collective dynamics of smoking in a large social network. N Engl J Med. 2008;358(21):2249-2258.

18. Cobb NK, Graham AL, Abrams DB. Social network structure of a large online community for smoking cessation. Am J Public Health. 2010;100(7):1282-1289.

19. Cole-Lewis H, Perotte A, Galica K, et al. Social network behavior and engagement within a smoking cessation Facebook page. J Med Internet Res. 2016;18(8):e205.

20. Graham AL, Zhao K, Papandonatos GD, et al. A prospective examination of online social network dynamics and smoking cessation. PLoS One. 2017;12(8):e0183655.

21. Shi X, Adamic LA, Strauss MJ. Networks of strong ties. Physica A. 2007;378(1):33-47.

22. Panek E, Hollenbach C, Yang J, Rhodes T. The effects of group size and time on the formation of online communities: evidence from reddit. Social Media + Society. 2018;4(4):2056305118815908.

23. Thrul J, Belohlavek A, Hambrick D, Kaur M, Ramo DE. Conducting online focus groups on Facebook to inform health behavior change interventions: two case studies and lessons learned. Internet Interv. 2017;9:106-111.

24. Prochaska JO, DiClemente CC. Stages and processes of self-change of smoking: toward an integrative model of change. J Consult Clin Psychol. 1983;51(3):390-395.

25. Patrick DL, Cheadle A, Thompson DC, et al. The validity of selfreported smoking: a review and meta-analysis. Am J Public Health. 1994;84(7):1086-1093.

26. Thrul J, Meacham MC, Ramo DE. A novel and remote biochemical verification method of smoking abstinence: predictors of participant compliance. Tob Prev Cessat. 2018;4(May):20.

27. Fiore MC, Jaén CR, Baker TB, et al. A clinical practice guideline for treating tobacco use and dependence: 2008 update: a U.S. Public Health Service Report. Am J Prev Med. 2008;35(2):158-176.

28. Wasserman S, Faust K. Social Network Analysis: Methods and Applications. Cambridge, UK: Cambridge University Press; 1994.

29. Stead LF, Carroll AJ, Lancaster T. Group behaviour therapy programmes for smoking cessation. Cochrane Database Syst Rev. 2017;3:CD001007.

30. Balmford J, Borland R, Burney S. Is contemplation a separate stage of change to precontemplation? Int J Behav Med. 2008;15(2):141-148. 